\title{
Frequency Decay through Electromagnetic Radiation Absorption and Re-Emission by Inter-Galactic Dark Matter as an Alternate Explanation for the Hubble Constant
}

\author{
David Grant Taylor \\ Edmonton, Canada \\ Email: dgtaylor@telusplanet.net
}

Received 27 April 2016; accepted 21 June 2016; published 26 June 2016

Copyright (C) 2016 by author and Scientific Research Publishing Inc.

This work is licensed under the Creative Commons Attribution International License (CC BY).

http://creativecommons.org/licenses/by/4.0/

(c) (i) Open Access

\section{Abstract}

There is an alternate cause for the decay rate defined by Edwin Hubble's Cosmological Constant Theory. It can be proposed because inward motion is observed in the local Galaxies||Star groups around the Milky Way. The recession velocity of Galaxies farther out of is reasoned entirely from the increasing redshift in the frequency. The smaller the image of observed Galaxy/Cluster objects, the greater the downward shift in frequency of all Electro-Magnetic signals [EM]. An alternate cause for that downward shift could be through the absorption and re-emission through matter, leading to the absorption of some fraction of the energy quanta. There is nowhere in our Local Universe that is both absolutely devoid of matter and the continual formation of objects of all scales. If redshift was because of space expansion, it would increase the distance that signal had to travel. So a signal from GN-z11 stellar structure at 13.4 billion light years [LY] would take 13.4 billion years to travel. Assuming 13.8 billion years since the Big Bang would mean GN-z11 object travelled 13.4 billion $L Y$ in $\mathbf{4 0 0}$ million years. A current value for the Hubble constant is: $\mathrm{H}_{0}=(67.8 \pm 0.77) \mathrm{km} \cdot \mathrm{s}^{-1} \cdot \mathrm{Mpc}^{-1}$ a frequency is shift of $67.8 / \mathrm{c}$ over a single Mpc. An alternate expression would be a shift factor $2.261560 \mathrm{E}-5$ over a distance of $3.08567 \mathrm{E} 22 \mathrm{~m}$ or a redshift of 7.32923E-28 over a metre because of passage through a medium. Dark matter is a currently accepted phenomenon. It is proposed that properties include redshift's all normal matters that are put upon EM||Boson signals at the fraction stated above. The signal reduction|| frequency distortion happens at a quantum level for each occurrence, and so is not detectable until passage through millions of LY of Dark Matter. Support for this alternate supposition is reasoned from the fact that the M31 Galaxy and the NGC 300 Galaxy are at distances inconsistent with their Hubble recession velocity. 


\section{Keywords}

\section{Big Bang Theory, Dark Matter, EM Frequency Decay, GN-z11 Body, Density ||Volume|| Age Universe, Origin CMBR, Comic Egg, Infinite, Milky Way [MW], Andromeda Galaxy [M31]}

\section{Introduction}

This paper contests the cause of the Hubble Constant $\left[\mathrm{H}_{0}\right]$. Electromagnetic radiation from Galactic/Cluster objects as their distance from the Milky Way [MW] increases is seen as evidence of Hubble velocity away from the Local||MW Galactic cluster. The velocity is proportional to the distance from the same in Mega-Parsecs

$\left[D_{M P}\right]$ as is defined in the equation:

$$
\mathrm{V}_{\text {Hubble }}=\mathrm{H}_{0} * \mathrm{D}_{\mathrm{MP}}
$$

The Hubble constant decay rate is so miniscule; a reasonable proposition for that frequency reduction of the signal could be due to interference by inter-Galactic gases. Objects on the edge of the Universe (an example being theGN-z11 at 1.34000E10 [1] LY |4.0164829100E3) are measured to have a Doppler redshift of 11.1 [1]. A Doppler redshift exceeding 1.0 could indicate the velocity of the emitting object greater than transmission velocity of the signal. An alternate explanation would be the EM signal is distorted by Relativistic effects. This paper argues against the velocity of universe expansion demanded by the Big Bang theory with currently accepted times for that event, and signal transmission time from observed objects moving between the objects and our observation points.

An alternate cause for the EM frequency shifts downward would be Dark Matter throughout the Universe. There is also the ongoing formation of Stellar||Stellar grouping objects that evidences the existence of normal matter. Dark matter or normal matter particle absorbs, then re-emits that EM at a lower frequency and signal strength and so distorts the signal. That signal reduction||frequency distortion would happen at a quantum level for each occurrence and so not detectable until after passage through millions of light years [LY] of passage through Dark Matter.

When an atom absorbs a wave train (photon), the total momentum is conserved and therefore must appear within the atom. The electron is therefore accelerated due to the momentum transfer from the photon to the mobile electron. The much smaller fraction of momentum imparted to the nucleus is much smaller and can be neglected here. The electron is then accelerated during a length of time equal to the time of coherence of the absorbed radiation. After absorption, the energy stays in the atom for a short interval of time that explains the apparent reduced speed of light in gases. Finally, the mechanism is reversed and the energy absorbed of the wave is re-emitted [2].

Support for the alternate supposition in this paper is that in current Hubble Theory, the M31 Galaxy and the NGC 300 Galaxy are at distances inconsistent with their recession velocity.

\section{Calculated Decay Rate of Inter-Galactic Radiation in Metres}

The widely accepted values used for the following calculations are theoretically presumed exact to ten decimal places as illustrative presumptions; the thesis of this paper does not rely on exact values. Mass of the Universe is given as $1.0 \mathrm{E} 52 \mathrm{Kg}$ in matter and 1.0E53kg in Energy, a total of $1.10 \mathrm{E} 53 \mathrm{~kg}$. There is no current agreement on the age of the Universe. A central value from varied sources is used to argue the invalidity of those estimations. What is below begins a naming of variables and cites assumed physics constants.

$$
\begin{gathered}
\text { Light speed }=|\mathrm{c}|=299792458 \mathrm{mps} \\
\text { Metres per Second }=\mathrm{mps} \\
\text { Kilometres per Second }=\mathrm{kps} \\
\text { Gravitational Constant }=\mathrm{G}=6.6738400000 \mathrm{E}-11 \mathrm{~m}^{3} \cdot \mathrm{kg} \cdot \mathrm{s}^{2} \quad[3] \\
\mathrm{Pi}=3.1415900000 \mathrm{E} 0 \mathrm{~m} \\
\text { Year }_{\text {Sec }}=365.25 \times 24 \times 3600=3.1557600000 \mathrm{E} 7 \mathrm{~s}
\end{gathered}
$$




$$
\begin{aligned}
& \text { Planck Length }=\mathrm{PL}=1.61622290000 \mathrm{E}-35 \mathrm{~m} \quad[3] \\
& \text { Light Year }=\mathrm{LY}=9.4607304726 \mathrm{E} 15 \mathrm{~m} \quad \text { [3] } \\
& \text { Parsec }=\mathrm{pc}=3.0856780000 \mathrm{E} 16 \mathrm{~m} \text { [3] } \\
& \text { Megaparsec }=\mathrm{Mpc}=(3.0856780000 \mathrm{E}+16 \times 1.000 \sim 00 \mathrm{E} 6) \mathrm{m}=3.0856780000 \mathrm{E} 22 \mathrm{~m} \\
& \text { Mega Parsec in Light Year }=\mathrm{Mpc}_{\mathrm{LY}}=3.2615642195 \mathrm{E} 07 \text { [3] } \\
& \text { Universe Total Matter }=\operatorname{Univ}_{\text {Matter }}=1.00 \sim 00 \mathrm{E}+52 \mathrm{~kg} \\
& \text { Universe Total Energy }=\text { Univ }_{\text {Energy }}=1.00 \sim 00 \mathrm{E}+53 \mathrm{~kg} \\
& \text { Universe Total Mass }=\text { Univ }_{\text {Mass }}=1.100 \sim 00 \mathrm{E}+53 \mathrm{~kg} \\
& \text { Univ }_{\text {Age }}=\mathrm{BB}=13.8 \text { Billion years }=1.3800 \sim 00 \mathrm{E} 10 \text { years [4] } \\
& \text { Universe Age Seconds }=\operatorname{Univ}_{\mathrm{Sec}}=\operatorname{Univ}_{\mathrm{Age}} * \mathrm{Year}_{\mathrm{Sec}}=4.3549488000 \mathrm{E} 17
\end{aligned}
$$$$
\text { Hubble }_{\text {Constant }}=\mathrm{H}_{0}=67.77 \mathrm{~km} \cdot \mathrm{s}^{-1} \cdot \mathrm{Mpc}^{-1}=6.77700 \sim 00 \mathrm{E}+4 \mathrm{~m} \cdot \mathrm{s}^{-1} \cdot \mathrm{Mpc}^{-1} \quad \text { [5] }
$$

That the Hubble Constant value was from the European Space Agency's Planck 2013 Mission report does assert some validity. But data on the Constant has varied so much in the entire history of it observation, it is extremely unlikely we will ever have it to a Planck level certainty. But it is a valid logic presumption.

The GN-z11 compact Galaxy is currently the furthest observed object in our Cosmos. The 11.1 Redshift of GN-z11 [6] indicates a |1.3400 00E10 LY [ GN-z11 Dist_LY $\left._{\text {] }}\right]$ 4.10846E9|[pc]||4.10846E3 Mpc distance-[ GN-z11 $1_{\text {Dist_Mpc }}$ ] that is challengeable, but theoretically acceptable. A 13.4 Billion year|LY's old GN-z11 means our observations are 400 million years post BB formation

GN-z11 meter distance [ GN-z11 $\left.1_{\text {Dist_m }}\right]$ :

$$
\begin{aligned}
& \mathrm{GN}-\mathrm{z} 11_{\text {Dist_m }}=\mathrm{GN-z11_{ \text {Dist_LY } }} * \mathrm{LY} \mathrm{D}_{\text {Distance }} \\
& \text { GN-z11 } 1_{\text {Dist_m }}=1.3400 \sim 00 \mathrm{E} 10 \mathrm{LY} * 9.4607304726 \mathrm{E} 15 \mathrm{~m} / \mathrm{LY} \\
& \mathrm{GN-z11} 1_{\text {Dist_m }}=1.2677378833 \mathrm{E} 26 \mathrm{~m}
\end{aligned}
$$

GN-z11 distance in Mpc [ GN-z11 $\left.1_{\text {Dist_Mpc }}\right]$

$$
\begin{aligned}
\text { GN-z11 } & \text { Dist_Mpc }=G N-z 11_{\text {Dist_m }} / \mathrm{Mpc} \\
\text { GN-z11 } & =1.2677378833 \mathrm{E} 26 \mathrm{~m} / 3.0856780000 \mathrm{E} 22 \mathrm{~m} / \mathrm{Mpc} \\
\mathrm{GN}-\mathrm{z} 11_{\text {Dist_Mpc }} & =4.1084581195 \mathrm{E} 3 \mathrm{Mpc}
\end{aligned}
$$

Assuming Hubble constant, GN-z11 moved out at [ GN-z11 ${ }_{\text {но }}$ ]

$$
\begin{aligned}
\text { GN-z11 } & =\mathrm{H}_{0} * \mathrm{GN}-\mathrm{z} 11_{\text {Dist_Mpc }} \\
\mathrm{GN}-\mathrm{z} 11_{\mathrm{H} 0} & =6.777 \mathrm{E}+4(\mathrm{~m} / \mathrm{s}) / \mathrm{Mpc} * 4.1084581195 \mathrm{E} 3 \mathrm{Mpc} \\
\mathrm{GN}-\mathrm{z} 11_{\mathrm{H} 0} & =2.7843020676 \mathrm{E} 8 \mathrm{~m} / \mathrm{s}
\end{aligned}
$$

The Universe Volume [ Univ $_{\mathrm{Vol}}$ ]

$$
\begin{aligned}
\operatorname{Univ}_{\mathrm{Vol}} & =4 / 3 * \mathrm{pi} *\left(\mathrm{GN}-\mathrm{z} 11_{\text {Dist_m }}\right)^{3} \\
\mathrm{Univ}_{\mathrm{Vol}} & =4 / 3 * 3.1415900 \sim 00 \mathrm{E} 0 *(1.2677378833 \mathrm{E} 26 \mathrm{~m})^{3} \\
\mathrm{Univ}_{\mathrm{Vol}} & =8.5344790065 \mathrm{E} 78 \mathrm{~m}^{3}
\end{aligned}
$$

The Universe Energy/Matter density: 


$$
\begin{aligned}
& \operatorname{Univ}_{\text {Density }}=\operatorname{Univ}_{\text {Mass }} / \mathrm{Univ}_{\text {Vol }} \\
& \operatorname{Univ}_{\text {Density }}=1.100 \sim 00 \mathrm{E}+53 \mathrm{~kg} / 8.5344790065 \mathrm{E} 78 \mathrm{~m}^{3} \\
& \operatorname{Univ}_{\text {Density }}=1.2888894555 \mathrm{E}-26 \mathrm{~kg} / \mathrm{m}^{3}
\end{aligned}
$$

Light||EM signals move at $|c|$. If EM we observe moved beyond the velocity of c, most of Relativity, most of modern Physics and its constants would be invalid.

So the minimum time the EM signal returned [ GN-z11 EM_Return $_{\text {] }}$ would be:

$$
\begin{aligned}
& \mathrm{GN}-\mathrm{z} 11_{\text {EM_Return }}=\mathrm{GN}-\mathrm{z} 11_{\text {Dist_m }} / \mathrm{C} \\
& \text { GN-Z11 } 1_{\text {EM_Return }}=1.2677378833 E 26 \mathrm{~m} / 299792458 \mathrm{~m} / \mathrm{s}
\end{aligned}
$$

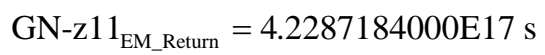

The time in years GN-z11 moved out [ GN-z11 $\left.1_{\text {Yrsout }}\right]$ would be the presumed age of the Universe, less the light year time for the returning EM.

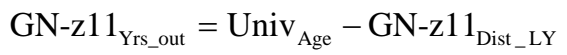

$$
\begin{aligned}
& \mathrm{GN}_{\mathrm{Z}} 11_{\text {Yrs_out }}=1.3800 \sim 00 \mathrm{E}+10 \text { years }-1.3400 \sim 00 \mathrm{E}+10 \text { years } \\
& \text { GN-z11 } 1_{\text {Yrs_out }}=4.00 \sim 00 \mathrm{E}+8 \text { years }
\end{aligned}
$$

In GN_z11 time out in seconds_-GN-z11 Sec_out $_{\text {_out }}$

$$
\begin{aligned}
& \text { GN-z11 } 1_{\text {Sec_Out }}=\text { Year }_{\text {Seconds }} * \text { GN-z11 } 1_{\text {Time_Outward_Years }} \\
& \text { GN-z11 } 1_{\text {Sec_Out }}=3.1557600000 \mathrm{E} 7 \mathrm{~s} / \mathrm{yr} * 4.00 \sim 00 \mathrm{E}+8 \mathrm{yr} \\
& \text { GN-z11 } 1_{\text {Sec_Out }}=1.2623040000 \mathrm{E} 16 \mathrm{~s}
\end{aligned}
$$

The velocity outward of GN-z11 [GN-z11 $1_{\text {Vel_out }}$ ]

$$
\begin{aligned}
\mathrm{GN}-\mathrm{z} 11_{\text {Vel_Out }} & =\mathrm{GN}-\mathrm{z} 11_{\text {Dist_m }} / \mathrm{GN}-\mathrm{z} 11_{\text {Sec_Out }} \\
\mathrm{GN}-\mathrm{z} 11_{\text {Vel_Out }} & =1.2677378833 \mathrm{E} 26 \mathrm{~m} / 1.2623040000 \mathrm{E} 16 \mathrm{~s} \\
\mathrm{GN}-\mathrm{z} 11_{\text {Vel_Out }} & =1.0043047343 \mathrm{E} 10 \mathrm{~m} / \mathrm{s}
\end{aligned}
$$

As a multiple of $|c|$ :

$$
\begin{aligned}
\mathrm{GN}-z 11_{\text {Vel_Out_c }} & =\mathrm{GN}-\mathrm{z} 11_{\text {Vel_Out }} / \mathrm{c} \\
\mathrm{GN}-\mathrm{z} 11_{\text {Vel_Out_c }} & =(1.0043047343 \mathrm{E} 10 \mathrm{~m} / \mathrm{s}) / 2.99792458 \mathrm{~m} / \mathrm{s} \\
\mathrm{GN}-\mathrm{z} 11_{\text {Vel_Out_c }} & =3.35000 \mathrm{E} 1
\end{aligned}
$$

Some theories propose the GN-z11's distortion is spatial expansions. That velocity/spatial expansion drops below $|c|$ as we develop the technology to observe it. Stretching space would distort the frequency as well. We would observe GN-z11's frequency distortion to be 33.5*c. The returning signal would travel the medium at $|c|$. The travel distance for the light would increase as it moved. Expanding space would stretch/shift the EM signal; appearing to exceed $|c|$. So the postulated beginning Universe was a mass compacted into a quantum dimension sphere expanding at a hyper-light velocity. GN-z11's velocity/expansion has slowed just as we observe it to below $|\mathrm{c}|$, increasing beyond " $\mathrm{c}$ " to an infinitely expanding Universe-expanding at ultra high and low densities. Infinitely expanding Universes are logically invalid. And no postulates on the formation of Universe's beginning—who/what laid the Cosmic Egg.

Illustrating with a Quantum $\|$ Planck $_{\text {Length }}$ diameter Cosmic Egg volume: :

$$
\begin{aligned}
& \text { Cosmic_Egg }_{\text {Volume }}=4 / 3 * \Pi *\left(\text { Planck }_{\text {Length }} / 2\right)^{3} \\
& \text { Cosmic_Egg }_{\text {Volume }}=4 / 3 * 3.1415926536 *(1.6162290000 \mathrm{E}-35 \mathrm{~m} / 2)^{3} \\
& \text { Cosmic_Egg }_{\text {Volume }}=2.2105854514 \mathrm{E}-105 \mathrm{~kg} / \mathrm{m}^{3}
\end{aligned}
$$


Divided into its mass:

$$
\begin{aligned}
& \text { Cosmic_Egg } \text { Density }_{\text {Universe }}=\text { Uass }_{\text {Unic }} / \text { Egg }_{\text {Volume }}
\end{aligned}
$$

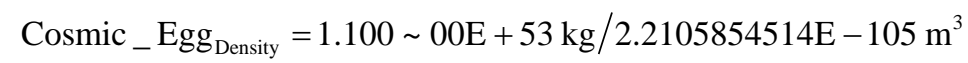

$$
\begin{aligned}
& \text { Cosmic_Egg } \text { Density }=4.9760573577 \mathrm{E} 157 \mathrm{~kg} / \mathrm{m}^{3}
\end{aligned}
$$

So current Science argues the Universe expanding at an average rate of $1.00430 \mathrm{E} 10 \mathrm{~m} / \mathrm{s}$ with densities ranging from $4.9760573577 \mathrm{E} 157 \mathrm{~kg} / \mathrm{m}^{3}$ to $1.2888894555 \mathrm{E}-26 \mathrm{~kg} / \mathrm{m}^{3}$. By the Hubble constant, slowing to $2.8479831684 \mathrm{E} 8 \mathrm{~m} / \mathrm{s}$ when reaching GN-z11's 13.4 billion LY distance.

The current measured redshift of GN-z11 is 11.1 [1]. Above calculations reason a 33.5* average velocity. If declining to $11.1^{*} \mathrm{C}$ now, the space expansion in front of the signal widened above $|\mathrm{c}|$ throughout. Signals emitting at the moment of the BB would never return; the space in front of that signal would have widened at pace greater than c. Spatial expansion and the distance of GN-z11 are mutually exclusive propositions. A single Boson $|c|$ particle moving through an expanding space will appear to have moved away greater than $|c|$. But the expansion of space will mean that distance between any point and GN-z11 will have increased. There is no evidence of spatial expansion other than interpretations that come with current estimates for the age of our Local Universe. Even assuming spatial expansion, the space in front of any Boson expands as does the space behind, so it still travels 13.4 Billion LY. Because of the certainty of Boson velocity, an expansion moving out objects at hyper-relativistic velocities would still take 13.4 Billion years to return. We see the GN-z11 object as it was 13.4 billion years ago. Adding a hyper-Relativistic expansion to $11.1^{*} \mathrm{C}$ expansion now would mean that the signal would never reach us, because the space in front of light speed Bosons would be increasing at a greater pace than they were moving

The above presumes the signal moved through an absolute vacuum, despite the ongoing dynamic formation of stellar||Galactic objects throughout our reality. If an EM signal/Photon impacts matter objects ranging from quantum scales upward, some energy transfers to the matter object. The CMB is estimated the same as a gas at approximately 3K [7]-[9]. The CMB presumes EM from an unobserved event. Hubble theory presumes Redshift of objects millions||billions of LY away can only be explained by motion. They presume a Universe expanding at a hyper-Relativistic pace through matter/energy densities ranging from $1.34030 \mathrm{E} 158 \mathrm{~kg} / \mathrm{m}^{3}$ to $1.2888894555 \mathrm{E}-26$ $\mathrm{kg} / \mathrm{m}^{3}$. If an aspect of a theory is indeterminate, how valid is it? An alternate postulate for the CMB and the Hubble constant does not need exceptions to Relativity and Space coherence.

Objects at the GN-z11 distance scale appear to be beginning, infant Galactic objects. But EM decay defined by the Hubble constant would eventually have a distortive effect on the signal. An article written on the body's discovery: "The primordial galaxy that they found is so remote that its EM is detectable only at the longest infrared wavelengths that Hubble can see" [10]. Limits to observable EM of an object mean the true image no longer exists.

\section{Relativistically Slowed EM/Boson Transmission}

Returning signals from objects on the edge of the Local [Observed] Universe would not move at $|c|$. Universe expansion or high Relativistic velocity means a signal moves at a slower pace were there no relativistic distortion. At any single point light velocity would be measured as “c”. Expansion would increase the signal travel distance. From an experimental viewpoint, were a signal were released, adding the expansion to the distance defined in the experiment would mean the signal travelled a greater distance-the distance travelled to a predefined object would increase. So calculate the velocity GN-z11 recedes at in kilometres per second [kps]

$$
\begin{aligned}
\mathrm{GN}-\mathrm{z} 11_{\text {Recede_kps }} & =\mathrm{GN}-\mathrm{z} 11_{-} \text {Dist }_{\mathrm{Mpc}} * \mathrm{H}_{0} \\
\mathrm{GN}-\mathrm{z} 11_{\text {Recede_kps }} & =4.1084581195 \mathrm{E} 3 \mathrm{Mpc} * 67.77[\mathrm{~km} / \mathrm{s}] / \mathrm{Mpc} \\
\mathrm{GN}-\mathrm{z} 11_{\text {Recede_kps }} & =2.7843020676 \mathrm{E} 5 \mathrm{~km} / \mathrm{s}
\end{aligned}
$$

And in metres per second [mps]

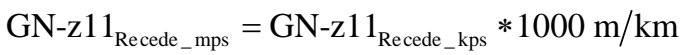

$$
\begin{aligned}
& \mathrm{GN}-\mathrm{z} 11_{\text {Recede_mps }}=(2.7843020676 \mathrm{E} 5 \mathrm{~km} / \mathrm{s}) *(1000 \mathrm{~m} / \mathrm{km}) \\
& \mathrm{GN}-\mathrm{z} 11_{\text {Recede_mps }}=2.7843020676 \mathrm{E} 8 \mathrm{~m} / \mathrm{s}
\end{aligned}
$$


Presume the object simply travelled at a Hubble Constant velocity. Relativistic Time distortion [Relativistic_Dist] would slow photon velocity and increase the Redshift [ GN-z11 $\left.1_{\text {Relativistic_Dist }}\right]$ :

$$
\begin{aligned}
& \text { GN-z11 } 1_{\text {Relativistic_Dist }}=1 /\left(1-G N-z 11_{\text {Recede_mps Withdrawl_Velocity_m/s }}^{2} / \mathrm{c}^{2}\right)^{0.5} \\
& \text { GN-z11 }{ }_{\text {Relativistic_Dist }}=1 /\left(1-2.6974266148 \mathrm{E} 8^{2} / 299792458^{2}\right)^{0.5} \\
& \text { GN-z11 }{ }_{\text {Relativistic_Dist }}=2.6974266148 E 0
\end{aligned}
$$

That Relativistic distortion would slow any EM signal emanating from that body, increasing the redshift by the same proportion:

$$
\begin{aligned}
& \text { GN-z11 } 1_{\text {Relativistic_Dist_v }}=\mathrm{GN}-\mathrm{z} 11_{\text {Recede_mps }} * \mathrm{GN}-\mathrm{z} 11_{\text {Relativistic_Dist }} \\
& \mathrm{GN}-\mathrm{z} 11_{\text {Relativistic_Dist_v }}=2.7843020676 \mathrm{E} 8 \mathrm{~m} / \mathrm{s} * 2.6974266148 \mathrm{E} 0 \\
& \mathrm{GN}-\mathrm{z} 11_{\text {Relativistic_Dist_v }}=7.5104505008 \mathrm{E} 8 \mathrm{~m} / \mathrm{s}
\end{aligned}
$$

So by two arguments, by current physics presumptions, the GN-z11 object appears to be moving faster than $|c|$ in an extremely vacuous environment. It is established that EM signals move at the velocity of "c", 299792 $458 \mathrm{~m} / \mathrm{s}$ in a vacuum environment. Current observation interpretation contradicts this fundamental in modern science. Is it possible that the Hubble Shift is being misinterpreted? Alternately, that the Universe is much older than 13.8 Billion years? Or both?

Though that is not the fundamental logic failing of current theory. The current Big Bang postulate proposes an expansion of linear dimensions at both hyper-dense states and at the hyper-diffuse state that exists now. How then could a Cosmic Egg ever form?

Spatial expansion is not the only cause of observed frequency shift. Simple EM passages through a medium will redshift the wavelength. The simple redshift of a setting Sun confirms this. Upon interruption by an aspect of that medium, a new signal is generated. Part of the signal would raise the temperature whatever particle of the medium it collided with-generating another signal, but at lower frequency because of energy absorption.

\section{EM Absorption by Inter-Galactic Gases/Matter}

There is hard evidence of the existence of Dark Matter throughout our reality-the evidence being EM frequency shifts downward. The matter absorbs the radiation and increase their molecular/atomic level velocity emitting new photons; photons with a tiny reduction in their intensity and frequency.

Additionally, intergalactic spatial Matter may contribute to Red Shift observed in inter-Galactic distances, by a factor in numerous technical and non-technical publications. A summary of those publications is made in the NASA site: “NASA’s Hubble Confirms That Galaxies Are the Ultimate Recyclers” [11].

Astronomers believe that the color and shape of a galaxy is largely controlled by gas flowing through an extended halo around it. The above article effectively argues against inter-galactic space being a simple and absolute vacuum. An inevitable consequence of any EM signal passing through disperse gas is the frequency shift because of signal transmission through any medium. It is unreasonable to suggest that the mass of Galactic\| Sub-Galactic [G|SG] disperse matter simply ends at the "Halos" surrounding them. The Hubble telescope produced a "halo of gas is shown with a radius of about 300,000 LY, although it may extend significantly further" [12] — significantly further than the one that is reasoned to surround the MW.

There would also be a much more disperse overwhelmingly Hydrogen gas Cosmosphere universally. An imaginary "Ether" substance is not needed. The gravity of G|SG objects would never be strong enough or direct enough for permanent capture of all of that Cosmosphere. There is a titanic variation in the mass and breadth in all cosmic objects. There is universal variation in the rate \& vectors of the movement of those objects.

Growth and evolution of all Stellar||Stellar group formation is an ongoing process throughout the Universe. So effectively, matter is everywhere. We only observe matter concentrated enough to substantially generate, divert or reflect/absorb EM in a substantial enough fashion to be visible from hundreds, thousands, or millions of Parsecs away. Its density will be debated endlessly.

But its existence can be declared as an absolute. It also offers additional components to CMB: simple baryon matter in the form of a superheated interstellar||intergalactic gas, at a temperature of a million degrees. The gas 
permeates the upper atmosphere of our planet, transferring its low magnitude energy to Earth’s atmosphere. That magnitude and the extremely low frequency of the signal would mean stabilizing at the CMB value. Frequency decline would also diffract (a currently incalculable) amount-a more direct explanation for the Baryon composition of the CMB.

A more reasonable deduction from current observations than invention of dark energy from evidence millions/billions of LY/pc away exists. The collective EM radiation from the entire Universe would be extremely dispersed. It would be the simple radiation from the 3K matter/Cosmosphere, in an environment just short of the vacuum absolute. So it is argued that the CMB is not a leftover from the Big Bang but a simple demonstration of the "temperature" of our reality. Dark matter is not a confirmed phenomenon. Dr. Christian Corda suggests an alternate:

In this different context, we are not required to find candidates for dark energy and dark matter-which till now have not been found-but only the "observed” ingredients, which are curvature and baryon matter, have to be taken into account [13].

Radiation from the entire Cosmos travels through the extremely disperse Cosmosphere of our Local Universe. As each photon collides with individual matter particles of all sizes, having the effect of increasing particle velocity-new Photons emerging at lesser frequency and intensity. The collective action of all free particles in a given density would lead to a relatively smooth radiation-a radiation level determined by collective EM sources surrounding any point.

Observed CMB level is for our limited immediate Space||Time region. It is unreasonable to assert that level to all of reality, because of a moderately homogenous level from an infinitesimal viewing point in our Local Universe (our planet; Solar system). An inter-galactic Cosmosphere would have the effect of putting a tiny red shift on the parent signal. At a particularly tiny quantum level for each exchange, but the effect would accumulate at the Parsec, the Mega-Parsec level. The current value for the CMB was reported in The Astrophysical Journal:

The FIRAS data are independently recalibrated using the WMAP data to obtain a CMB temperature of $2.7260+/-0.0013$. Measurements of the temperature of the cosmic microwave background are reviewed. The determination from the measurements from the literature is cosmic microwave background temperature of $2.72548+/-0.00057 \mathrm{~K}$ [14].

There was additional evidence for this supposition recently: “The Boomerang Nebula: The Coldest Region of the Universe?” [15]. The Nebula was observed to be within a cosmologically crisp one degree Kelvin. While the expansion of the gas would cool it, it would also greatly expand its exposure to the CMB. The nebula is presumed to be at a pre-planetary stage. So the centre point of the Nebula would be its densest and warmest part of the body. There would also be the continual input of the core itself. It is not a gas expansion happening in carefully structured and blocked cooling pipes; it is happening in an area surrounded by EM radiation from both within and without. There is also the simple temperature maintenance structure that the CMB represents. If the $\mathrm{CMB}$ is the Universal phenomenon it is touted to be, the Boomerang Nebula could never descend to its present temperature. It would cool to the point where it was the same temperature as the surrounding environment. It is arguable that the CMB is not of a great enough magnitude to "re-heat" any cooled object. It is unreasonable to suppose that any mass of matter would lose more energy as it compressed such that its temperature would fall below the ambient temperature around it. Objects heat up as they compress-if that fundamental is denied, then almost all theory of stellar/Galactic formation would have to be dismissed.

A parallel, very alternate proposal to this explanation was made in a paper called An Explanation for the Hubble Constant in a Static Universe [16]. It does accept the redshift through matter cloud diffraction, though it limits the nature of the phenomenon to be pure electron clouds, a phenomenon never documented in our reality. There is also another paper in which it the Hydrogen spectrum to the CMB is cited as evidence for the matter cloud to be principally that element [17] that would invalidate that explanation.

Another shortcoming to current theory is that we cannot declare absolutely that the CMB is the same throughout our reality_it was first discovered by Arno Penzias and Robert Wilson in June of 1963 [18]. Declaring a phenomenon from 53 years of observation from a sphere with the same value LY radius as an absolute declaration for the condition of the Universe is invalid. Our Local Universe has dimensions that are on a scale of billions of years/LY's so data is incomplete for a scientific declaration of its validity. 


\section{Inconsistent Galactic Velocities}

Another argument can be made: the Andromeda Galaxy [M31] is currently estimated to be 7.8500 - 00E2 kiloparsec (7.8500 - 00E-1 Mpc [ M31 ${ }_{\mathrm{Mpc}}$ ] or 2.5600 - 00E6LY [ M31 $1_{\mathrm{LY}}$ ]) [19] away. The Hubble Constant $\left[\mathrm{H}_{0}\right.$ ] recession velocity [ M31 $1_{\text {Recess_v }}$ ]:

$$
\begin{aligned}
& \text { M31 } 1_{\text {Recess_v }}=\mathrm{M} 31_{\mathrm{Mpc}} * \mathrm{H}_{0} \\
& \mathrm{M} 31_{\text {Reces__v }}=7.8500 \sim 00 \mathrm{E}-1 \mathrm{Mpc} * 6.77700 \sim 00 \mathrm{E}+1 \mathrm{kps} / \mathrm{Mpc} \\
& \mathrm{M} 31_{\text {Recess_v }}=5.3199450000 \mathrm{E} 1 \mathrm{kps}
\end{aligned}
$$

M31's is current measured velocity, $301 \pm 1 \mathrm{~km} / \mathrm{s}$ [20] [ M31 ], is towards the MW. The two bodies may have been separating in the early moments of the Universe. But the mass of M31 is thought to equivalent to that of the MW, 1.0E12 Solar Masses [ $\mathrm{MW}_{\text {Solar_Mass }}$ ].The mass of the Sun [ $\mathrm{Sun}_{\text {Mass }}$ ] is estimated to be $1.0 \mathrm{E} 30 \mathrm{~kg}$ [21]. So both Galaxies mass approximately:

$$
\begin{aligned}
& \mathrm{MW}_{\text {Mass }}=\operatorname{Sun}_{\text {Mass }} * \mathrm{MW}_{\text {Solar_Mass }} \\
& \mathrm{MW}_{\text {Mass }}=1.0 \mathrm{E} 30 * 1.0 \mathrm{E} 12 \\
& \mathrm{MW}_{\text {Mass }}=1.0 \mathrm{E} 42 \mathrm{~kg}
\end{aligned}
$$

The distance between M31 and MW in metres is:

$$
\begin{aligned}
& \mathrm{M} 31_{\text {Dist_m }}=\mathrm{LY}_{\text {Dist }} * \mathrm{M} 31_{\mathrm{LY}} \\
& \mathrm{M} 31_{\text {Dist_m }}=9.4607304726 \mathrm{E}+15 \mathrm{~m} / \mathrm{LY} * 2.5600 \sim 00 \mathrm{E}+6 \mathrm{LY} \\
& \mathrm{M} 31_{\text {Dist_m }}=2.4219470010 \mathrm{E} 22 \mathrm{~m}
\end{aligned}
$$

The Escape velocity between the two bodies:

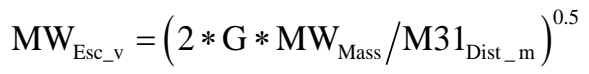

$$
\begin{aligned}
& \mathrm{MW}_{\text {Esc_v }}=(2 * 6.67384 \mathrm{E}-11 * 1.0 \mathrm{E} 36 \mathrm{~kg} / 2.42194 \mathrm{E} 22 \mathrm{~m})^{0.5} \\
& \mathrm{MW}_{\mathrm{Esc} \_\mathrm{v}}=1.17056 \mathrm{E} 5 \mathrm{~m} / \mathrm{s}
\end{aligned}
$$

And the proportion of the actual velocity to escape velocity would be

$$
\begin{aligned}
\text { Prop }_{\text {EscV_to_V }}=\mathrm{MW}_{\text {Esc_V }} /{\mathrm{M} 31_{\mathrm{V}}} \\
\text { Prop }_{\text {Esc__to_V }}=3.01000 \mathrm{E} 5 \mathrm{~m} / \mathrm{s} / 1.17056 \mathrm{E} 5 \mathrm{~m} / \mathrm{s} \\
\text { Prop }_{\text {Esc__to_V }}=2.57142 \mathrm{E} 0
\end{aligned}
$$

So M31 is approaching the MW at more than $2 \frac{1}{2}$ times their mutual escape velocity, in an expanding Universe. The escape velocity would be less than the above. The escape equation uses the distance between both objects centre of gravity. But both objects are so disperse, that the centre of gravity is a debatable issue. The above is the absolute maximum escape velocity; they may be mutually approaching at a much greater proportion. Is that not another argument for frequency decay because of the Local Universe Cosmosphere as an explanation as opposed to a Hubble Reality? M31 is simply moving towards us at a velocity too high to be overcome by Cosmosphere frequency shift.

This paper will not speculate as to the true age of the Universe. Later papers will examine that age. But a minimum age for the Universe can be proposed. The first question is: when did the density of the Universe reach the point where a hyper-light velocity was no longer reasonable? That point is neither in current theory or available from the objections made in this paper.

But let us propose that the reason that the M31 body is approaching us and not receding is that it rests at the limit of how far there could be spontaneous expansion. So what would the density of a homogenous sphere with containing the entire assumed matter/energy mass of the Universe-a sphere with a radius equal to the distance between the MW and M31. Because M31 is approaching the MW now, it would have been farther away at the time. The below is only a theoretic tool to reason the validity/invalidity of the Big Bang conjecture. The volume of such a sphere [ MW || M31 Sphere_Volume $_{\text {] }}$ ] would be: 


$$
\begin{aligned}
& \text { MW } \| \text { M31 } 1_{\text {Sphere_Volume }}=4 / 3 * 3.14159 *\left(\mathrm{M}_{1} 1_{\text {Dist_m }}\right)^{3} \\
& \text { MW } \| \text { M31 } 1_{\text {Sphere_Volume }}=4 / 3 * 3.14159 *(2.4219470010 \mathrm{E} 22 \mathrm{~m})^{3} \\
& \text { MW } \| \text { M31 } 1_{\text {Sphere_Volume }}=2.83761 \mathrm{E} 67 \mathrm{~m}^{3}
\end{aligned}
$$

So the density of such a sphere would then be the Mass of the Universe ( Universe $_{\text {Mass }}$ ) divided by the volume of the sphere.

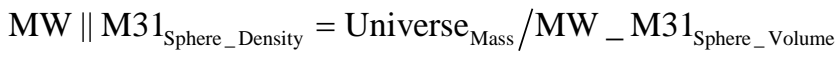

$$
\begin{aligned}
& \text { MW } \| \text { M31 } 1_{\text {Sphere_Density }}=1.10000 \mathrm{E}+53 \mathrm{~kg} / 2.83761 \mathrm{E} 67 \mathrm{~m}^{3} \\
& \text { MW } \| \text { M31 } 1_{\text {Sphere_Density }}=3.52409 \mathrm{E}-15 \mathrm{~kg} / \mathrm{m}^{3}
\end{aligned}
$$

And its gravitational force [GF]

$$
\begin{aligned}
& \mathrm{MW} \| \mathrm{M} 31_{\text {Sphere_Gravity }}=\text { GMass }_{\text {Universe }} /\left(\mathrm{M} 31_{\text {Distance_Metres }}\right)^{2} \\
& \mathrm{MW} \| \mathrm{M} 31_{\text {Sphere_Gravity }}=6.67384 \mathrm{E}-11 \mathrm{~m}^{3} /\left(\mathrm{kg} \cdot \mathrm{s}^{2}\right) * 1.10000 \mathrm{E}+53 \mathrm{~kg} /(2.421947 \mathrm{E} 22 \mathrm{~m})^{2} \\
& \mathrm{MW} \| \mathrm{M} 31_{\text {Sphere_Gravity }}=1.864088 \mathrm{E}-3 \mathrm{~m} / \mathrm{s}^{2}
\end{aligned}
$$

Surely it is unreasonable to suppose that a sphere with a density of $3.52409 \mathrm{E}-15 \mathrm{~kg} / \mathrm{m}^{3}$ and a Gravity of $1.864088 \mathrm{E}-3 \mathrm{~m} / \mathrm{s}^{2}$ would be sufficient to force Hyper-EM velocity expansion of the Universe.

Presume it took 3.8 Billion years for the MW\|M31 group to form. Also presuming the average velocity to be one-half what it is now-to calculate arithmetically would be possible, but that would require knowledge of the distorting effects of all secondary, tertiary, quaternary, et al. bodies in the MW $\mid \mathrm{M} 31$ Cluster.

So 10 billion years ago, the distance of M31 would be its position now PLUS whatever distance it has travelled since then. "Escape velocity" is not the maximum velocity that could be imposed on an approaching object. Escape velocity is where the kinetic energy of an object were it launched is equal to the potential energy of an object were it at an infinite distance - the definition presumes that the end velocity to be zero. A projectile object moving at well beyond escape velocity will still have its outward velocity lessened by gravity. But its outward velocity would not be Parabolic; it would be Hyperbolic - never decreasing to zero. In parallel, the velocity of projectile object approaching a gravitational body would be determined by its approaching velocity as well as the velocity imposed by the gravity of the object. Gravitational acceleration would not cease until the approaching body reached |c|; something impossible in our reality. The interfering gravitational effect of the other bodies in our local group makes a true mathematic reasoning for the average approach speed impossible. We also cannot know the full vectors of the approach velocity; we only observe the vector directly aimed at our observation point. So presume the initial approaching velocity ( $\mathrm{M} 1_{\mathrm{Avg}_{-} \text {Approach_V }}$ ) was half what is observed now.

$$
\begin{aligned}
& \mathrm{M} 31_{\text {Avg_Approach_V }}=\mathrm{M} 31_{\text {Velocity }} / 2 \\
& \mathrm{M} 31_{\text {Avg_Approach_V }}=(3.0100 \mathrm{E} 5 \mathrm{~m} / \mathrm{s}) / 2 \\
& \mathrm{M} 31_{\text {Avg_Approach_V }}=1.50500 \mathrm{E} 5 \mathrm{~m} / \mathrm{s}
\end{aligned}
$$

The distance of M31 ten Billion years ago [ M31_ Dist ${ }_{10 \_B i l l i o n \_Y e a r s}$ ] would be that velocity distance added to its current distance in space:

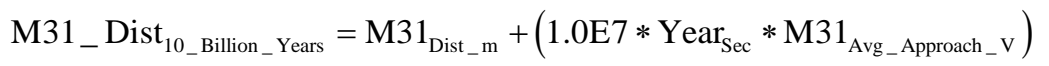

$$
\begin{aligned}
& \text { M31__Dist }{ }_{10 \_B i l l i o n \_Y e a r s}=2.42194 \mathrm{E} 22 \mathrm{~m}+(1.0 \mathrm{E} 7 * 3.15576 \mathrm{E} 7 \mathrm{~s} / \mathrm{yr} * 1.50500 \mathrm{E} 5 \mathrm{~m} / \mathrm{s}) \\
& \text { M31_Dist }{ }_{10 \_B i l l i o n \_Y e a r s}=6.64156 E 22 \mathrm{~m}
\end{aligned}
$$

That same distance in light years

$$
\begin{aligned}
& \text { M31_Dist_LY } 10_{\text {10Billion_Yrs_Ago }}=\text { M31_Dist } \text { 10_Billion_Yrs_Ago }_{\text {Ago }} / \mathrm{LY}_{\text {Distance }}
\end{aligned}
$$

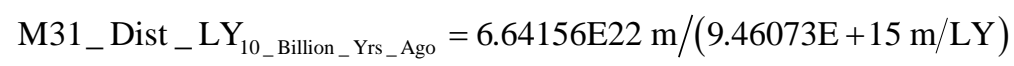

$$
\begin{aligned}
& \text { M31_Dist_LY } 10_{\text {_Billion_Yrs__Ago }}=7.02014 \mathrm{E} 6 \text { LY }
\end{aligned}
$$

There is a Galaxy at approximately that distance: NGC 300. Its distance now is 6.85E+6 LY [22]. Its redshift 
is what it would be for an object moving at $1.45 \mathrm{E} 5 \mathrm{~m} / \mathrm{s}$. That velocity is less than half of M31, but in an absolutely opposite vector. That would mean that 10 Billion years ago, M31 was at the approximate distance of NGC 300. The distance of NGC 300 in metres now is

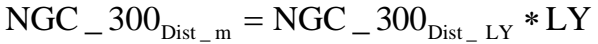

$$
\begin{aligned}
& \text { NGC_ } 300_{\text {Dist_m }}=6.85 \mathrm{E}+6 \mathrm{LY} * 9.46073 \mathrm{E}+15 \mathrm{~m} / \mathrm{LY} \\
& \text { NGC_300 }{ }_{\text {Dist_m }}=6.47997 \mathrm{E} 22 \mathrm{~m}
\end{aligned}
$$

According to the principle of the Hubble constant, we will presume the current velocity of NGC 300 velocity is above what it was 10 Billion years ago-so presume its average velocity [ NGC_300 Avg_Recede_v $]$ since then was only 0.5 that: $7.25 \mathrm{E} 4 \mathrm{~m} / \mathrm{s}$.

Its distance then would be its current distance less its movement outward [ NGC_300 Dist_10_Billion_Ago $]$ since then:

$$
\begin{aligned}
& \text { NGC_300 } \text { Dist_10_Billion_Ago }=\mathrm{NGC} \_300_{\text {Distance }_{-} \mathrm{m}}-\left(1.0 \mathrm{E} 10 * \mathrm{Year}_{\mathrm{Sec}} * \mathrm{NGC} \_300_{\text {Avg_Recede_V }_{-}}\right) \\
& \text {NGC_300 } \text { Dist_10_Billion_Ago }=6.47997 \mathrm{E} 22 \mathrm{~m}-(1.0 \mathrm{E} 10 \mathrm{yr} * 3.15576 \mathrm{E} 7 \mathrm{~s} / \mathrm{yr} * 7.25000 \mathrm{E} \mathrm{m} / \mathrm{s}) \\
& \text { NGC_300 } \text { Dist_10_Billion_Ago }=6.479967 \mathrm{E} 22 \mathrm{~m}-2.28793 \mathrm{E} 22 \mathrm{~m} \\
& \text { NGC_300 } \text { Dist_10_Billion_Ago }=4.19204 \mathrm{E} 22 \mathrm{~m}
\end{aligned}
$$

That distance in Light Years:

$$
\begin{aligned}
& \text { NGC_300 } \text { Dist_10_Billion_Ago_LY }=\mathrm{NGC} \_300_{\text {Dist_10_Billion_Ago }} / \mathrm{LY} \\
& \text { NGC_300 } \\
& \text { NGist_10_Billion_Ago_LY } \\
& \text { NGC_300 } \\
& \text { Dist_10_Billion_Ago_LY }
\end{aligned}
$$

So current theory approximates that 10 billion years ago NGC 300 was 4.43099E6LY away and M31 was 7.02014E6LY. In that time the two of them have moved millions of LY in opposite directions. Does this not demonstrate irretrievably that the Hubble Constant||Universe Expansion is a more complex issue than simple recession velocity as a ratio of distance? Is frequency decay through EM absorption by free, extremely disperse gaseous matter a valid alternate to a theory that has absolutely conflicting data items in its hypothesis?

\section{Summary}

The discovery of the Hubble Constant || $\mathrm{H}_{\mathrm{o}}$ was first made by Georges Lemaître in 1927 and confirmed by Edwin Hubble in 1929. Since then it has been taken as uncontestable evidence by some in science as the initial detonation of all our reality, labeled the Big Bang. A very absolute majority currently accepts that un-contestability now, but that does not increase its validity in logic. The principle evidence given for the supposition is that the Red Shift that forms the base for that constant could only come about through increasing velocity as the body's separation from the Earth increases. There are strong arguments against this proposal. The principle one would be that the expansion the Universe would then have proceeded for a hyper-Boson velocity for its entire lifetime. That would not lead to a form that was the dimensions and nature of our Universe. A valid alternate explanation would be a Redshift brought about with the absorption/reemission by inter-galactic dark matter lessening the EM frequency at a rate of 7.32924E-27/m. Additional arguments are reasoned for this evidence in following papers, with equations derived directly from the Classic Relativity Equations. The fundamental point in this entire paper can be expressed very simply: which is the most reasonable supposition? The Universe is not a perfect vacuum - established in many studies. The interference of an extreme disperse Cosmosphere of gaseous matter diffracts and redshifts all EM radiation entering into it. It would be at such a slow rate because of the diffuseness of the gas. The redshift would make it appear the farther an object it, the greater the rate of recession. Alternately, current theory maintains a Universe level mass of matter/energy 13.8 billion years ago spontaneously exploded. That mass expanded at an average 20 times the speed of light but under dispersion at that pace was spontaneously able to form stellar/cluster/Galactic matter objects?

This alternate supposition gains additional argument from the fact that by current Hubble theory, the M31 Galaxy and the NGC 300 Galaxy are at distances inconsistent with their recession velocity. 


\section{References}

[1] Hubble Team Breaks Cosmic Distance Record.

http://www.nasa.gov/feature/goddard/2016/hubble-team-breaks-cosmic-distance-record

[2] Marmet, P. (1988) A New Non-Doppler Redshift. Physics Essays, 1, $24-32$.

http://www.newtonphysics.on.ca/hubble/index.html

[3] NIST Reference. http://physics.nist.gov

[4] Harrington, J.D. and Whitney, C. Planck Mission Brings Universe into Sharp Focus. NASA News Releases. http://www.nasa.gov/home/hqnews/2013/mar/HQ_13-079_Planck_Mission.html

[5] Planck Collaboration (2013) Planck 2013 Results. I. Overview of Products and Scientific Results. Astronomy \& Astrophysics Manuscript: Planck Mission 2013. http://arxiv.org/pdf/1303.5062v2.pdf

[6] A New Record: Keck Observatory Measures Most Distant Galaxy. http://astronomynow.com/2015/08/06/a-new-record-keck-observatory-measures-most-distant-galaxy/

[7] The Origins of the Universe: The Cosmic Microwave Background. http://www.ctc.cam.ac.uk/outreach/origins/cmb.php

[8] Universe 101: Discovery of the Cosmic Microwave Background. http://map.gsfc.nasa.gov/universe/bb tests cmb.html

[9] The Cosmic Microwave Background. Department of Astronomy and Astrophysics, Penn State University, University Park. https://www.e-education.psu.edu/astro801/content/110 p8.html

[10] Mann, A. (2011) Oldest Galaxy Is Lone Ranger. Nature. http://www.nature.com/news/2011/110126/full/news.2011.47.html

[11] Hubble Space Telescope. NASA’s Hubble Confirms That Galaxies Are the Ultimate Recyclers. http://www.nasa.gov/mission_pages/hubble/science/recyclers.html

[12] Hubble Space Telescope . Chandra: The Milky Way’s Hot Gas Halo. http://www.nasa.gov/mission_pages/chandra/multimedia/hot_gas_halo.html

[13] Interferometric Detection of Gravitational Waves: The Definitive Test for General Relativity. International Journal of Modern Physics D, 18, 2275-2282. http://www.worldscientific.com/doi/pdf/10.1142/S0218271809015904

[14] Fixsen, D.J. (2009) The Temperature of Cosmic Microwave Background. The Astrophysical Journal, 707, $916-920$. http://iopscience.iop.org/0004-637X/707/2/916/

[15] Sahai, P. and Nyman, L.-A. (2009) The Boomerang Nebula: The Coldest Region of the Universe? The Astrophysical Journal Letters, 487, 916-920. http://iopscience.iop.org/1538-4357/487/2/L155/

[16] An Alternate Explanation for the Hubble Constant. Proceedings of the NPA. https://www.academia.edu/6615695/An explanation of redshifts in a static universe

[17] Brandenberger, R.H. (1995) Formation of Structure in the Universe. http://arxiv.org/pdf/astro-ph/9508159v2.pdf

[18] Discovery of the Cosmic Microwave Background. American Physical Society, Physics. https://www.aps.org/publications/apsnews/200207/history.cfm

[19] McConnachie, A.W., Irwin, M.J., Ferguson, A.M.N., Ibata, R.A., Lewis, G.F. and Tanvir, N. (2005) Distances and Metallicities for 17 Local Group Galaxies. Monthly Notices of the Royal Astronomic Society, 356, 979-997. http://mnras.oxfordjournals.org/content/356/3/979.full?sid=1a570508-7670-42a8-af78-b4879a80e7cb

[20] Karachentsev, I.D. and Kashibadze, O.G. (2006) Masses of the Local Group and of the M81 Group Estimated from Distortions in the Local Velocity Field. Astrophysics, 49, 3-1.8 http://link.springer.com/article/10.1007\%2Fs10511-006-0002-6

[21] Williams, D.R. Sun Fact Sheet. http://nssdc.gsfc.nasa.gov/planetary/factsheet/sunfact.html

[22] Blair, W.P. and Long, K.S. (1997) Identification of Supernova Remnants in the Sculptor Group Galaxies NGC 300 and NGC 7793. The Astrophysical Supplement Series, 108, 261-277. http://iopscience.iop.org/0067-0049/108/1/261/fulltext/ 